\title{
CONVECTION IN MODERATELY HIGH CONCENTRATED COLLOIDAL SUSPENSIONS
}

\author{
M. DarAssi \\ Princess Sumaya University for Technology \\ P.O. Box 1438 Al-Jubaiha \\ Amman, 11941, JORDAN
}

\begin{abstract}
A particulate medium model is considered. The viscosity is assumed to depend on concentration through the general empirical formula. The onset of convection in moderately high concentrated colloidal suspensions layer is investigated and the threshold conditions for the convection onset are obtained. A weakly nonlinear stability analysis is conducted and a nonlinear evolution partial differential equation is derived. The Poincaré - Lindstedt method is applied to obtain a uniformly valid periodic solution to the sought evolution equation. A full asymptotic analysis is performed and our findings is compared with the results obtained by different models. Some numerical simulations are presented.
\end{abstract}

AMS Subject Classification: 76E06, 35Q35, 76D05

Key Words: convection, colloidal suspension, evolution equation, periodic solution

\section{Introduction}

Convection in colloidal suspensions is one of the most important processes in many recent industries [1], [2]. Heat transfer in colloidal suspensions occurs through both diffusion and convection. Convection in colloidal suspensions is theoretically investigated using the same mathematical formalism as convection in binary mixture. However, convection in colloidal suspensions is characterized by larger particle size, smaller Lewis number and larger separation ratio.

$\begin{array}{lr}\text { Received: } & \text { April 24, } 2017 \\ \text { Revised: } & \text { July 18, 2017 } \\ \text { Published: } & \text { August 9, 2017 }\end{array}$

(c) 2017 Academic Publications, Ltd. url: www.acadpubl.eu 
Since the characteristics of Soret-driven have been understood, most of the results obtained through the use of thermosolutal convection equations for binary mixture are extended to the case of colloidal suspensions [3]-[7]. Recent experimental work has demonstrated that the addition of a small volume fraction of particles may enhance the thermal conductivity of the fluid [8]-[10].

The addition of small particles transfer the convection in colloidal suspension into a very complicated multi-scale problem. These scales are thermal diffusion scale, particle diffusion scale, thermophoresis scale and sedimentation scale. The viscosity is assumed to depend on particle concentration through the general empirical formula $\bar{\mu}=\mu_{0}\left(1-C / C_{M}\right)^{-2}$ where $\bar{\mu}$ is the dynamic viscosity and $C_{M}$ is the maximum packing volume fraction for hard-sphere colloids. The particulate medium model [11] is considered because both particle size and particle volume fraction are depicted in the mathematical model. Hence it is the best to model convection in thermosensitive colloids [3]. The situation where the coefficient of thermal expansion is assumed zero has been considered in [12] to investigate the onset of particle-dominated regime. The case where both viscosity and the coefficient of particle diffusion depend linearly on concentration through Einstein's formula is discussed in details in[13]. The linear stability of the onset of convection in moderately high concentrated colloidal suspension has been investigated in [14].

The parameter $\beta=\beta_{T}-\beta_{S}$ is introduced by Chang et. al. which depicts the effects of sedimentation and thermophoresis where $\beta_{T}$ is the Soret coefficient and $\beta_{S}$ is the sedimentation Péclet number. This parameter is a function of the particle radius which has approximately inverted parabola shape with two zero crossings. The first zero crossing corresponds to the particles of molecular size which models binary mixtures. The second zero crossing corresponds to particles of larger size although still in nanosize range which represents the nearly balanced effects of sedimentation and thermophoresis [15]. We considered a layer of a moderately high concentrated colloidal suspension which is confined between two parallel horizontal plates and heated from below. We investigated the linear stability and determined the threshold conditions for the onset of convection using the particulate medium model in [14]. In this work we extended the work done in [14] to weakly nonlinear stability analysis. Also we extended the work done in [13] to beyond dilute limit case.

Investigation of liquid suspension is difficult even in the absence of heating. Several conservation equations and empirical formulas are combined in the mathematical model to describe the liquid suspensions. In the dilute limit case the particles hydrodynamic interactions are neglected [16]. Tokuyama and Oppenheim [17] presented a systematic theory for the dynamics of concentrated 
hard-sphere suspensions of particles interactions with both direct and hydrodynamic interactions. They put forth the role of the short and long range hydrodynamic interactions played in the dynamic of concentrated hard-sphere suspensions. In [18], Tokuyama and Oppenheim investigated the effects of the hydrodynamic and direct interactions of the particles in the dynamics of hardsphere suspensions. Due to the hydrodynamic interactions, they showed that there are two many-body effects, the static (Screening) effect and the dynamic (Correction) effect. Others have included the thermal diffusion effects. For instance, Dhont ([19], [20]) used a microscopic approach to study the theory of the inter colloidal interactions in the presence of thermal diffusion. Ning et.al.[21] conducted experiments to verify the theory of colloidal suspension interactions at low concentration. In the majority of theoretical studies that consider thermal diffusion in dilute systems, the interactions between the colloid particles are neglected. In this paper we consider the case of low concentrated hard-sphere suspension. We aim at extending results that we have obtained in the dilute concentration limit, $\Phi_{m} \approx 2-3 \%$ to concentration levels up to about $\Phi_{m} \approx 10 \%$ while maintaining the assumptions of negligible particle-particle interactions. This assumption is actually supported by experimental work in Refs. [16], [21].

This paper is organized as follows. In Section 2, a mathematical model of mass dominated convection regime in nanofluid is introduced. In Section 3, we make use of the long-wavelength asymptotic to derive an evolution equation for the leading-order value of the particle concentration valid near the instability threshold. A full analysis of the evolution equation is undertaken for the sake of determining a valid uniformly period solution in Section 4. The findings obtained through the solution of the particulate medium formulation are compared with those obtained using the mathematical formalism of binary convection and some numerical simulations are presented in Section 5.

\section{Mathematical Model}

We consider a moderately high concentrated colloidal suspension layer that is confined between two rigid, perfectly conducting and impermeable to mass-flow plates. The plates located at $Z=0$ and $Z=H$, where $Z$ is the vertical coordinate. The bottom plate maintained at temperature $T_{0}$ and the top plate at $T_{1}$. The viscosity is assumed to depend on the particles concentration through the general empirical formula $\bar{\mu}=\mu_{0}\left(1-C / C_{M}\right)^{-2}$, where $\bar{\mu}$ is the dynamic viscosity and $C_{M}=0.64$ is the maximum packing volume fraction for hardsphere colloids. The Boussinesq approximation is applied i.e. the density is 
constant everywhere in the governing equations except in the gravity term in the momentum equation it depends on both temperature and concentration [22], namely

$$
\rho=\rho_{0}\left(1+\gamma_{S} C+\gamma_{T}\left(T-T_{0}\right)\right)
$$

where $\rho$ is the fluid density, $\rho_{0}$ is the density of the fluid at the reference temperature $T_{0}, \gamma_{S}$ and $\gamma_{T}$ are the coefficients of thermal and solutal expansion, respectively. The non-dimensionalization process for the equations is as in [13]. The length is scaled by the the distance between the two plates $H$, the time by the characteristic time for thermal diffusion, $H^{2} / \kappa$, the temperature by the temperature difference between the two plates, $\Delta T$ and the particle concentration by its maximum packing volume fraction value $C_{M}$. The concentration basic state profile is given by $C_{B}(z)=\lambda \exp (\beta z)$ and the temperature basic profile is given by $T_{B}(z)=-z$ and where $\lambda=\Phi_{m} \beta /\left(e^{\beta}-1\right)$. The viscosity will depend on $C_{B}(z)$ and is expressed in the general form as $\bar{\mu}=C_{M}^{2} F\left(C_{B}(z)\right)$ where $F\left(C_{B}(z)\right)=\left(1-C_{B}\right)^{-2}$.

Upon subtracting the basic state profiles, we obtain the non-dimensional system describing the conservation equations of mass, momentum, energy and particles concentration for the convective quantities,

$$
\begin{gathered}
\nabla \cdot \mathbf{u}=0 \\
\operatorname{Pr}^{-1}\left(\frac{\partial \mathbf{u}}{\partial t}+(\mathbf{u} \cdot \nabla) \mathbf{u}\right)=-\nabla p+\nabla \cdot(F \nabla \mathbf{u})+(R T-B C) \mathbf{k} \\
\frac{\partial T}{\partial t}+\frac{d T_{B}}{d z} \mathbf{u} \cdot \mathbf{k}+\mathbf{u} \cdot \nabla T=\nabla^{2} T \\
\tau^{-1}\left(\frac{\partial C}{\partial t}+\frac{d C_{B}}{d z} \mathbf{u} \cdot \mathbf{k}+\mathbf{u} \cdot \nabla C\right)=\nabla^{2} C-\beta \frac{d C}{d z} \\
+\beta_{T}\left[\left(C+C_{B}\right) \nabla^{2} T+\frac{d C_{B}}{d z} \frac{d T}{d z}+\nabla C \cdot \nabla T\right]
\end{gathered}
$$

Where $\beta$ is the dimensionless particles speed, $\operatorname{Pr}=\nu / \kappa$ the Prandtl number, $\tau=\mathcal{D} / \kappa$ the Lewis number, $S=\gamma_{C} C_{M} /\left(\gamma_{T} \Delta T\right)$ is the separation ratio where $\Delta T$ is the temperature difference between the two plates, and the two control parameters, namely

$$
R=\frac{\gamma_{T} \Delta T g H^{3} C_{M}^{2}}{\nu \kappa}, \quad B=R S
$$

are the Rayleigh number and the Buoyancy number, respectively. Where $\mathcal{D}$ is the thermal diffusion coefficient, $\mathrm{g}$ is the gravity, $\nu$ is the kinetic viscosity, 
$\kappa$ is the thermal diffusivity. The corresponding boundary conditions, which pertain to rigid, perfectly conducting and impermeable to mass-flow plates are described by

$$
\begin{gathered}
\mathbf{u}=\mathbf{0}, \quad \frac{\partial C}{\partial z}-\beta_{S} C+\beta_{T} \frac{\partial T}{\partial z} C=0 \quad \mathrm{z}=0,1 . \\
T=0, \quad \text { at } \mathrm{z}=0 \quad T=-1, \quad \text { at } \mathrm{z}=1
\end{gathered}
$$

\section{Weakly Nonlinear Stability Analysis}

Following the general procedure used by [23, 24] the equations (2) - (5) can be simplified by eliminating the continuity equation and using the general representation for the velocity vector field $\mathbf{u}=\nabla \times(\nabla \times \psi \mathbf{k})$, where $\psi$ is the poloidal component of the velocity vector field.

$$
\begin{gathered}
F \nabla^{4} \psi+2(D F) \nabla^{2}(D \psi)+\left(D^{2} F\right) D^{2} \psi=-B C+R \theta \\
\theta_{t}+\nabla_{H}^{2} \psi+(D \psi)_{x} \theta_{x}+(D \psi)_{y} \theta_{y}-\nabla_{H}^{2} \psi D \theta=\nabla^{2} \theta \\
\tau^{-1}\left(C_{t}+\lambda \beta \mathrm{e}^{\beta z} \nabla_{H}^{2} \psi+(D \psi)_{x} C_{x}+(D \psi)_{y} C_{y}-\nabla_{H}^{2} \psi D C\right) \\
=\nabla^{2} C-\beta D C+\beta_{T}\left[\left(C+\lambda \mathrm{e}^{\beta z}\right) \nabla^{2} \theta+\beta \lambda \mathrm{e}^{\beta z} D \theta+\nabla C \cdot \nabla \theta\right]
\end{gathered}
$$

subject to the boundary conditions $\psi(0)=\psi(1)=0, D \psi(0)=D \psi(1)=0$, $\theta(0)=\theta(1)=0$ and $D C-\beta C+\beta_{T} D \theta=0$ at $z=0,1$. Here $\mathbf{u}=\langle u, v, w\rangle$ $=<\psi_{x z}, \psi_{y z},-\nabla_{H}^{2} \psi>$ is the stream function representation of the velocity vector $\mathbf{u}$ and $\nabla_{H}^{2} \psi=\psi_{x x}+\psi_{y y}$. Upon using the transformation $C=\beta \mathrm{e}^{\beta z} \phi$, equations (7) - (9) reduced to

$$
\begin{gathered}
\mathrm{e}^{-\beta z}\left(1-\lambda \mathrm{e}^{\beta z}\right)^{-2} \nabla^{4} \psi+4 \lambda \beta\left(1-\lambda \mathrm{e}^{\beta z}\right)^{-3} \nabla^{2}(D \psi) \\
+\beta^{2}\left[6 \lambda^{2} \mathrm{e}^{\beta z}\left(1-\lambda \mathrm{e}^{\beta z}\right)^{-4}+2 \lambda\left(1-\lambda \mathrm{e}^{\beta z}\right)^{-3}\right] D^{2} \psi=-\tilde{B} \phi+R \mathrm{e}^{-\beta z} \theta \\
\theta_{t}+\nabla_{H}^{2} \psi+(D \psi)_{x} \theta_{x}+(D \psi)_{y} \theta_{y}-\nabla_{H}^{2} \psi D \theta=\nabla^{2} \theta \\
\tau^{-1}\left(\phi_{t}-(\lambda+D \phi+\beta \phi) \nabla_{H}^{2} \psi+\phi_{x} D \psi_{x}+\phi_{y} D \psi_{y}\right) \\
=D^{2} \phi+\beta \phi+\nabla_{H}^{2} \phi+\beta_{T}\left[(\phi+\lambda / \beta) \nabla^{2} \theta+(\lambda+D \phi+\beta \phi) D \theta+\nabla_{H}^{2} \phi \cdot \nabla_{H}^{2} \theta\right]
\end{gathered}
$$


where $\tilde{B}=\beta B$. To derive a nonlinear evolution equation, we apply the long wavelength expansion analysis on the equations (10) - (12). We introduce the parameter $\epsilon, 0<\epsilon \ll 1$, and the slow variables $X=\epsilon x, Y=\epsilon y, \eta=$ $\epsilon^{4} t$. We expand the Rayleigh and buoyancy numbers as $R=R_{c}+\epsilon^{2} R_{2}$ and $\tilde{B}=\tilde{B}_{c}+\epsilon^{2} \tilde{B}_{2}$. Also the independent variables are expanded as follows: $\psi=$ $\psi_{0}+\epsilon^{2} \psi_{2}+\ldots, \theta=\theta_{0}+\epsilon^{2} \theta_{2}+\epsilon^{4} \theta_{4}+\ldots$ and $\phi=\phi_{0}+\epsilon^{2} \phi_{2}+\epsilon^{4} \phi_{4}+\ldots$ Since a small values of $\beta$ are considered, we scale $\beta_{T}=\epsilon^{2} \widehat{\beta}_{T}$ and $\beta=\epsilon^{2} \widehat{\beta}$, where $R_{2}, \tilde{B}_{2}, \widehat{\beta}_{T}$ and $\widehat{\beta}$ are $O(1)$ quantities. For simplicity we consider the one-dimensional problem. The $\mathrm{O}(1)$ problem is described by

$$
\begin{gathered}
\left(1+2 \Phi_{m}\right) D^{4} \psi_{0}=-\tilde{B}_{c} \phi_{0}+R_{c} \theta_{0} \\
D^{2} \theta_{0}=0 \\
D^{2} \phi_{0}+\left(\Phi_{m} \beta_{T} / \beta\right) D^{2} \theta_{0}=0
\end{gathered}
$$

Whose solution is given by

$$
\begin{gathered}
\psi_{0}=\frac{-\tilde{B}_{c} h}{24\left(1+2 \Phi_{m}\right)}\left(Z^{4}-2 Z^{3}+Z^{2}\right) \\
\theta_{0}=0 \quad \text { and } \quad \phi_{0}=h(X, \eta)
\end{gathered}
$$

Proceeding to the next order, the $O\left(\epsilon^{2}\right)$ the problem is described by

$$
\begin{gathered}
\left(1+2 \Phi_{m}\right) D^{4} \psi_{2}-\widehat{\beta}\left(Z+\Phi_{m}\right) D^{4} \psi_{0}+2\left(1+2 \Phi_{m}\right) D^{2}\left(\psi_{0}\right)_{X X} \\
+4 \Phi_{m}\left(1+3 \Phi_{m}\right) D^{3} \psi_{0}=-\tilde{B}_{c} \phi_{2}-\tilde{B}_{2} \phi_{0}+R_{c} \theta_{2}+R_{2} \theta_{0} \\
D^{2} \theta_{2}=\left(\psi_{0}\right)_{X X} \\
\tau^{-1}\left[\left(\phi_{0}\right)_{X}\left(D \psi_{0}\right)_{X}-\Phi_{m}\left(\psi_{0}\right)_{X X}\right]=D^{2} \phi_{2}+\left(\phi_{0}\right)_{X X}+\left(\Phi_{m} \beta_{T} / \beta\right) D^{2} \theta_{2}
\end{gathered}
$$

Application of the solvability condition, which is to integrate both sides of equation (20) from $Z=0$ to $Z=1$, yields the following expression for $\tilde{B}_{c}$

$$
\tilde{B}_{c}=\frac{720 \tau\left(1+2 \Phi_{m}\right)}{\Phi_{m}}
$$

Upon using the scaling $\tilde{B}_{c}=\beta B_{c}$ and the fact that $B_{c}=R_{c} S$, the critical Rayleigh number is given by

$$
R_{c}=\frac{720 \tau}{\mathcal{S}}\left(1+2 \Phi_{m}\right)
$$


where $\mathcal{S}=\beta \Phi_{m} S$. Which is consistent with the critical Rayleigh number value obtained in the linear theory in[14]. The solution of the $O\left(\epsilon^{2}\right)$ problem is given by

$$
\begin{gathered}
\psi_{2}=\frac{\tilde{B}_{c} \widehat{\beta} h}{120\left(1+2 \Phi_{m}\right)^{2}}\left(1+4 \phi_{m}\right) Z^{5}+\frac{\tilde{B}_{c} h_{X X}}{720\left(1+2 \Phi_{m}\right)}\left(3 Z^{6}-6 Z^{5}-10 Z^{4}\right) \\
+\frac{\tilde{B}_{c}^{2}\left(h_{X}\right)^{2}}{725760 \tau\left(1+2 \Phi_{m}\right)^{2}}\left(2 Z^{9}-9 Z^{8}+12 Z^{7}-21 Z^{4}\right) \\
+\frac{\Phi_{m} \tilde{B}_{c}^{2}\left(\widehat{\beta}_{T} / \widehat{\beta}+\tau^{-1}\right) h_{X X}}{7257600\left(1+2 \Phi_{m}\right)}\left(2 Z^{10}-10 Z^{9}+15 Z^{8}-60 Z^{4}\right) \\
-\frac{R_{c} \tilde{B}_{c} h_{X X}}{7257600\left(1+2 \Phi_{m}\right)^{2}}\left(2 Z^{10}-10 Z^{9}+15 Z^{8}-42 Z^{5}\right) \\
-\frac{\widehat{\mu}^{2} h}{24\left(1+2 \Phi_{m}\right)} Z^{4}+L_{1} Z^{3}+L_{2} Z^{2}
\end{gathered}
$$

where the constants $L_{1}$ and $L_{2}$ are given by

$$
\begin{gathered}
L_{1}=\frac{-3 \widehat{\beta} \tilde{B}_{c}\left(1+4 \Phi_{m}\right) h}{40\left(1+2 \Phi_{m}\right)^{2}}+\frac{22 \tau^{-1} \tilde{B}_{c}^{2}}{725760\left(1+2 \Phi_{m}\right)^{2}}\left(h_{X}\right)^{2}+\frac{26 \tilde{B}_{c}}{720\left(1+2 \Phi_{m}\right)} h_{X X} \\
-\frac{84 \Phi_{m} \tilde{B}_{c}^{2}\left(\widehat{\beta}_{T} / \widehat{\beta}+\tau^{-1}\right)}{7257600\left(1+2 \Phi_{m}\right)^{2}} h_{X X}-\frac{90 R_{c} \tilde{B}_{c}}{7257600\left(1+2 \Phi_{m}\right)^{2}} h_{X X}+\frac{\widehat{\mu}^{2} h}{12\left(1+2 \Phi_{m}\right)} \\
L_{2}=\frac{2 \widehat{\beta} \tilde{B}_{c}\left(1+4 \Phi_{m}\right) h}{40\left(1+2 \Phi_{m}\right)^{2}}-\frac{6 \tau^{-1} \tilde{B}_{c}^{2}}{725760\left(1+2 \Phi_{m}\right)^{2}}\left(h_{X}\right)^{2}-\frac{13 \tilde{B}_{c}}{720\left(1+2 \Phi_{m}\right)} h_{X X} \\
+\frac{31 \Phi_{m} \tilde{B}_{c}^{2}\left(\widehat{\beta}_{T} / \widehat{\beta}+\tau^{-1}\right)}{7257600\left(1+2 \Phi_{m}\right)^{2}} h_{X X}+\frac{55 R_{c} \tilde{B}_{c}}{7257600\left(1+2 \Phi_{m}\right)^{2}} h_{X X}-\frac{\widehat{\mu}^{2} h}{24\left(1+2 \Phi_{m}\right)} \\
\theta_{2}=\frac{-\tilde{B}_{c} h_{X X}}{1440\left(1+2 \Phi_{m}\right)}\left(2 Z^{6}-6 Z^{5}+5 Z^{4}-Z\right) \\
\phi_{2}=-\frac{\tau^{-1} \tilde{B}_{c}\left(h_{X}\right)^{2}}{1440\left(1+2 \Phi_{m}\right)}\left(12 Z^{5}-30 Z^{4}+20 Z^{3}-1\right) \\
+\frac{\Phi_{m} \tilde{B}_{c}^{2}\left(\widehat{\beta}_{T} / \widehat{\beta}+\tau^{-1}\right) h_{X X}}{10080\left(1+2 \Phi_{m}\right)}\left(14 Z^{6}-42 Z^{5}+35 Z^{4}-2\right)-\frac{\tilde{B}_{c} h_{X X}}{2}\left(Z^{2}-1\right)
\end{gathered}
$$


Proceeding to the order $O\left(\epsilon^{4}\right)$, we have

$$
\begin{aligned}
& D^{2} \phi_{4}+\left(\Phi_{m} \widehat{\beta}_{T} / \widehat{\beta}\right) D^{2} \theta_{4}= \\
& \tau^{-1}\left[\left(\phi_{0}\right)_{t}-\Phi_{m}\left(\psi_{2}\right)_{X X}+\left(\Phi_{m} \widehat{\beta} / 2-D \phi_{2}-\widehat{\beta} \phi_{0}\right)\left(\psi_{0}\right)_{X X}\right. \\
& \left.+\left(\phi_{0}\right)_{X}\left(D \psi_{2}\right)_{X}+\left(\phi_{2}\right)_{X}\left(D \psi_{0}\right)_{X}\right] \\
& -\widehat{\beta} D \phi_{2}-\left(\phi_{2}\right)_{X X}-\widehat{\beta}_{T}\left(D^{2} \theta_{2} \phi_{0}+\Phi_{m} D \theta_{2}-\left(\Phi_{m} / 2\right) D^{2} \theta_{2}\right) \\
& -\left(\Phi_{m} \widehat{\beta}_{T} / \widehat{\beta}\right)\left(\theta_{2}\right)_{X X} \text {. }
\end{aligned}
$$

Application of the solvability condition, that is integrate both sides of equation (26) for $\phi_{4}$ over the liquid layer thickness, yields the sought evolution equation

$$
h_{\eta}=-\mathcal{A} h_{X X X X}-\widehat{\mu}^{2} \mathcal{B} h_{X X}+\mathcal{C}\left(h_{X}^{3}\right)_{X}+\mathcal{E}\left(h_{X}^{2}\right)_{X X}+\mathcal{F}\left(h^{2}\right)_{X X}
$$

where

$$
\begin{gathered}
\mathcal{A}=\frac{\tau}{924}\left(34-131 \tau\left[\widehat{\beta}_{T} / \widehat{\beta}+(1 / \mathcal{S})\right]\right) \\
\mathcal{B}=\frac{\Phi_{m}}{720\left(1+2 \Phi_{m}\right)}, \quad \mathcal{C}=\frac{10 \tau}{\left(7 \Phi_{m}^{2}\right)} \quad \mathcal{E}=-\frac{\tau}{2 \Phi_{m}}+\frac{\left(\widehat{\beta}_{T} / \widehat{\beta}\right) \tau^{2}}{4 \Phi_{m}}
\end{gathered}
$$

and

$$
\mathcal{F}=-\tau \widehat{\beta} / 2 \Phi_{m}
$$

\section{Uniformly Valid Periodic Solution}

Following the general procedure of using the Biot number [29]. The term $-\widehat{\gamma} h$ is added to equation (27), to get

$$
h_{\eta}=-\mathcal{A} h_{X X X X}-\widehat{\mu}^{2} \mathcal{B} h_{X X}-\widehat{\gamma} h+\mathcal{C}\left(h_{X}^{3}\right)_{X}+\mathcal{E}\left(h_{X}^{2}\right)_{X X}+\mathcal{F}\left(h^{2}\right)_{X X}
$$

where

$$
\begin{gathered}
\mathcal{A}=\frac{\tau}{924}\left(34-131 \tau\left[\widehat{\beta}_{T} / \widehat{\beta}+(1 / \mathcal{S})\right]\right) \\
\mathcal{B}=\frac{\Phi_{m}}{720\left(1+2 \Phi_{m}\right)}, \quad \mathcal{C}=\frac{10 \tau}{\left(7 \Phi_{m}^{2}\right)} \quad \mathcal{E}=-\frac{\tau}{2 \Phi_{m}}+\frac{\left(\widehat{\beta}_{T} / \widehat{\beta}\right) \tau^{2}}{4 \Phi_{m}}
\end{gathered}
$$


and

$$
\mathcal{F}=-\tau \widehat{\beta} / 2 \Phi_{m}
$$

Upon using the following transformations

$$
h=a f, \quad \xi=b X, \quad \eta=e \widehat{t}, \quad \gamma=a \widehat{\gamma}
$$

and $\mu^{2}=\widehat{\mu}^{2} a b^{2} \mathcal{B} / 2$. Where

$$
a=\sqrt{\frac{\mathcal{A}}{\mathcal{C}}}, \quad b=\left(\frac{1}{\mathcal{A}} \sqrt{\frac{\mathcal{C}}{\mathcal{A}}}\right)^{1 / 4}, \quad e=1 / a
$$

we obtain

$$
f_{\widehat{t}}=-f_{\xi \xi \xi \xi}-2 \mu^{2} f_{\xi \xi}-\gamma f+\left(f_{\xi}^{3}\right)_{\xi}+\alpha_{1}\left(f_{\xi}^{2}\right)_{\xi \xi}+\alpha_{2}\left(f^{2}\right)_{\xi \xi}
$$

where

$$
\alpha_{1}=\frac{\mathcal{E}}{\sqrt{\mathcal{A C}}}, \quad \text { and } \quad \alpha_{2}=\mathcal{F} \sqrt[4]{\frac{\mathcal{A}}{\mathcal{C}^{3}}}
$$

To study the stability of the static solution of the equation (29), we will consider the linear part

$$
f_{\widehat{t}}=-f_{\xi \xi \xi \xi}-2 \mu^{2} f_{\xi \xi}-\gamma f
$$

Using the normal modes $f(\xi, \widehat{t})=\mathrm{e}^{\sigma \widehat{t}+i k \xi}$, we obtain the following dispersion relation

$$
\sigma=-\left(k^{2}-\mu^{2}\right)^{2}+\mu^{4}-\gamma
$$

The static state solution is unstable when $\gamma<\mu^{4}$. The weakly nonlinear stability of the evolution equation can be investigated by introducing the small parameter, $\epsilon \ll 1$, and conducting the perturbation analysis near the linear solution. We expand

$$
\begin{gathered}
\gamma=\mu^{4}-\epsilon \gamma_{1}-\epsilon^{2} \gamma_{2}, \quad \widehat{t}=\epsilon^{2} t \\
f=\epsilon f_{1}+\epsilon^{2} f_{2}+\epsilon^{3} f_{3}+\cdots
\end{gathered}
$$

The order $O(\epsilon)$ of equation (29) is described by

$$
\left(f_{1}\right)_{\xi \xi \xi \xi}+2 \mu^{2}\left(f_{1}\right)_{\xi \xi}+\mu^{4} f_{1}=0
$$

The periodic solution is given by $f_{1}=\cos (\mu \xi)$. Because of the secular terms, we will apply the Poincaré - Lindstedt method to obtain a uniformly valid 
periodic solution. Substitute $\nu=w \xi$ and expand $w=1+\epsilon w_{1}+\epsilon^{2} w_{2}+\cdots$ in equation (29) to obtain

$$
w^{4} f_{\nu \nu \nu \nu}+2 \mu^{2} w^{2} f_{\nu \nu}+\gamma f=w^{4}\left(f_{\nu}^{3}\right)_{\nu}+w^{4} \alpha_{1}\left(f_{\nu}^{2}\right)_{\nu \nu}+w^{2} \alpha_{2}\left(f^{2}\right)_{\nu \nu}
$$

The order $O(\epsilon)$ problem is described by

$$
\mathscr{L}\left(f_{1}\right)=0
$$

Where the above operator is defined by

$$
\mathscr{L}(f)=f_{\nu \nu \nu}+2 \mu^{2} f_{\nu \nu}+\mu^{4} f
$$

The solution of equation (34) is $f_{1}=\cos (\mu \nu)$. Proceeding to order $O\left(\epsilon^{2}\right)$ the problem is described by

$$
\mathscr{L}\left(f_{2}\right)=\gamma_{1} \cos (\mu \nu)+\left(2 \alpha_{1} \mu^{4}-\alpha_{2} \mu^{2}\right) \cos (2 \mu \nu)
$$

To remove the secular terms we set $\gamma_{1}=0$ which means that there is no subcritical instability. The solution of the resulting equation is given by $f_{2}=$ $\Gamma \cos (2 \mu \nu)$, where

$$
\Gamma=\frac{2}{9}\left(\alpha_{1}-\frac{\alpha_{2}}{\mu^{2}}\right)
$$

Proceeding to the next order $O\left(\epsilon^{3}\right)$ the problem described by

$$
\begin{gathered}
\mathscr{L}\left(f_{3}\right)=\left[\gamma_{2}-8 \mu^{4} w_{1}^{2}-(3 / 2) \Gamma \mu^{2} \alpha_{2}+3 \Gamma \mu^{4} \alpha_{1}-(1 / 4) \mu^{4}\right] \cos (\mu \nu) \\
\quad-w_{1}\left[2 \alpha_{2} \mu^{2}+48 \Gamma \mu^{4}-4 \alpha_{1} \mu^{4}\right] \cos (2 \mu \nu) \\
+\left[(1 / 4) \mu^{4}-(9 / 2) \Gamma \alpha_{2} \mu^{2}+9 \Gamma \alpha_{1} \mu^{4}\right] \cos (3 \mu \nu)
\end{gathered}
$$

To remove the secular term, we set $\gamma_{2}-8 \mu^{4} w_{1}^{2}-(3 / 2) \Gamma \mu^{2} \alpha_{2}+3 \Gamma \mu^{4} \alpha_{1}-$ $(1 / 4) \mu^{4}=0$ and hence solve for $w_{1}$ we get

$$
w_{1}=\sqrt{\frac{\gamma_{2}}{8 \mu^{4}}-\frac{1}{32}-\frac{3 \Gamma \alpha_{2}}{16 \mu^{2}}+\frac{3 \Gamma \alpha_{1}}{8}}
$$

The solution of equation (37) is given by

$$
f_{3}=Q_{1} \cos (2 \mu \nu)+Q_{2} \cos (3 \mu \nu)
$$

where

$$
Q_{1}=-(4 / 9) w_{1}\left[\frac{\alpha_{2}}{\mu^{2}}-2 \alpha_{1}+12 \Gamma\right]
$$


and

$$
Q_{2}=\frac{1}{256}-\frac{9 \Gamma \alpha_{2}}{128 \mu^{2}}+\frac{9 \Gamma \alpha_{1}}{64}
$$

Thus, the steady state solution of equation (29) is given by

$$
\begin{gathered}
f=\epsilon \cos \left(\mu\left(1+\epsilon w_{1}\right) \xi\right)+\epsilon^{2} \Gamma \cos \left(2 \mu\left(1+\epsilon w_{1}\right) \xi\right) \\
+\epsilon^{3}\left[Q_{1} \cos \left(2 \mu\left(1+\epsilon w_{1}\right) \xi\right)+Q_{2} \cos \left(3 \mu\left(1+\epsilon w_{1}\right) \xi\right)\right]
\end{gathered}
$$

The following graph depicted the uniformly valid periodic solution of the evolution equation (28) which is given by equation (39) with the parameters $\gamma_{2}=1, \epsilon=0.1, \tau=0.0001, \mathcal{S}=1, \Phi_{m}=0.01, \beta_{T}=0.8, \beta=0.5$ and $\mu=0.7$.

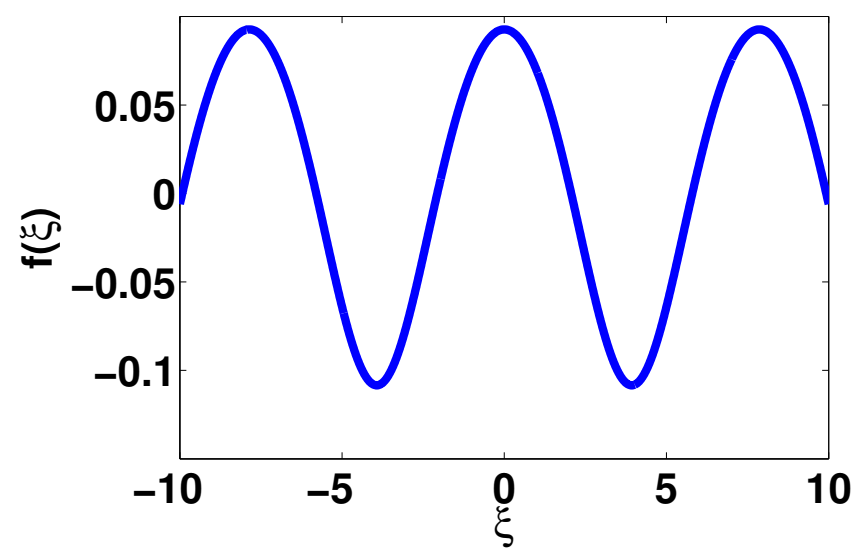

Figure 1: Plot of the periodic solution $f(\xi)$ with $\gamma_{2}=1, \epsilon=0.1, \tau=$ $0.0001, \mathcal{S}=1, \Phi_{m}=0.01, \beta_{T}=0.8, \beta=0.5$ and $\mu=0.7$

\section{Discussion and Conclusion}

Two horizontal rigid, completely conducting and impermeable to mass flow have been considered. The top and bottom plates maintained at temperature of $T_{1}$ and $T_{0}$, respectively. A moderately high concentrated colloidal suspension layer is confined between the two plates and heated from below. A particulate medium model has been adopted $[13,22]$. The particle diffusion, sedimentation and thermophoresis effects are depicted by the experimental parameter $\beta$ which is a function of the particle radius, $r_{p}$, with an approximately inverted parabola shape [15]. We have investigated the threshold conditions for the onset 
of the particle dominated convection regime in moderately high concentrated colloidal suspensions. our analysis is focused on the particle dominated convection regime, i.e. $0<\beta \ll 1$, which corresponds to positively large separation ratio case in the binary mixture model. The quasi-Boussinesq approximation has been applied. A strongly dependence of viscosity on particles' concentration has been assumed. This dependence is through the general empirical formula $\mu=\mu_{0}\left(1-C / C_{M}\right)^{-2}$.

Hadji and DarAssi in [13] conduct the long wave-length asymptotic analysis to derive the following analytical expression for the critical Rayleigh number, $\widehat{R}_{c}$, namely

$$
\widehat{R}_{c}=\frac{720 \tau\left(1+m \Phi_{m}\right)\left(1-a \Phi_{m}\right)}{\mathcal{S}\left[1+a \Phi_{m}(2-\gamma)+\left(1+a \Phi_{m} \gamma / 2\right)\left(\widehat{\beta}_{T} / \widehat{\beta}\right) \tau\right]}
$$

by assuming the linear dependence of both the dynamic viscosity, $\mu$, and the mass diffusion coefficient, $\mathcal{D}$, on the particles concentration as follows

$$
\mu=\mu_{0}\left(1+m \Phi_{m} C_{B}\right), \quad \mathcal{D}=D_{0}\left(1-a \Phi_{m} C_{B}\right)
$$

where $\gamma=\left(2 \beta_{T}+\beta_{S}\right) / \beta, m=2.5$, is the concentration coefficient in Einstein's equation, $a=6.55$ and $C_{B}$ is the concentration of the base state. For the sake of comparison, we will let $a=0$ (constant coefficient of mass diffusion $\mathcal{D}$ ) in equation (40) to obtain,

$$
\widehat{R}_{c}=\frac{720 \tau}{\mathcal{S}}\left(\frac{1+m \Phi_{m}}{1+\left(\widehat{\beta}_{T} / \widehat{\beta}\right) \tau}\right)
$$

where $\widehat{\beta}_{T}$ and $\widehat{\beta}$ are assumed to be $O(1)$ quantities and represent the thermophoretic and Stokes contribution to $\widehat{\beta}$, respectively and $\tau \ll 1$. Hence

$$
\widehat{R}_{c}=\frac{720 \tau}{\mathcal{S}}\left(1+m \Phi_{m}\right)+O\left(\tau^{2}\right)
$$

Alternatively, recall equation (22)

$$
R_{c}=\frac{720 \tau}{\mathcal{S}}\left(1+2 \Phi_{m}\right)
$$

Thus, the two expression equation (41) and equation (22) yield approximately the same expression for the critical Rayleigh number. It is noteworthy that equation (41) is derived for a dilute suspensions. However, equation (22) is derived for a moderately high concentrated suspensions. 
A long wave-length asymptotic expansion is carried out to derive the nonlinear evolution equation (27) which is analogous to the one derived in [26] using the binary mixture formalism. The evolution equation (27) is of a parabolic type, which is well posed if and only if the coefficient of the forth order derivative term is negative [25]. To obtain the validity region for which the asymptotic analysis is valid, we solve the inequality $\mathcal{A}>0$ for $\mathcal{S}$ to get

$$
\mathcal{S}>\frac{131 \tau}{34-131 \tau \widehat{\beta}_{T} / \widehat{\beta}}
$$

If the sedimentation effects are neglected, i.e. $\widehat{\beta}_{T}=\widehat{\beta}$, the well-known lower bound for the separation ratio $\mathcal{S}_{\infty}$ for long wave-length asymptotic expansion in a binary mixture model to be valid is retrieved [26], namely

$$
\mathcal{S}_{\infty}>\frac{131 \tau}{34-131 \tau}
$$

The validity regions where the asymptotic analysis used to derive the evolution equation (27), are depicted in figure (2), where the lower bound $\mathcal{S}_{\infty}$ is plotted versus $\widehat{\beta}$ for $\widehat{\beta}_{T}=0.1,0.5$ and 0.9 .

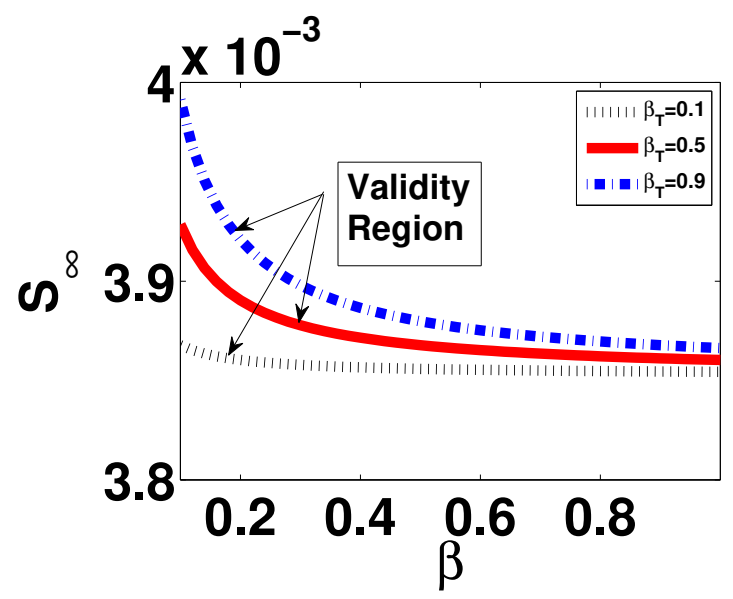

Figure 2: Plot of the lower bound $\mathcal{S}_{\infty}$ as a function of $\widehat{\beta}$ for $\widehat{\beta}_{T}=0.1$ (dots) line, $\widehat{\beta}_{T}=0.5$ (solid) line and $\widehat{\beta}_{T}=0.9$ (dashed) line.

In order to investigate the stability of the solution of the evolution equation (27), we first consider its linear part

$$
f_{\eta}=-\mathcal{A} h_{X X X X}-\mathcal{B} h_{X X}
$$


To derive the dispersion relation, we set $h=\exp (\sigma \eta+i k X)$, where $\sigma$ is the growth rate and $k$ is the wave-number. The following is the desired dispersion relation,

$$
\sigma=-\mathcal{A}\left(k^{2}-\frac{\mathcal{B}}{2 \mathcal{A}}\right)^{2}+\frac{\mathcal{B}^{2}}{4 \mathcal{A}}
$$

The plot of the growth rate $\sigma$ versus the square of the wave-number $k^{2}$ for different values of the mean volume fraction $\Phi_{m}$ is depicted in figure (3). A destabilizing effect is depicted with an increase of the values of $\Phi_{m}$.

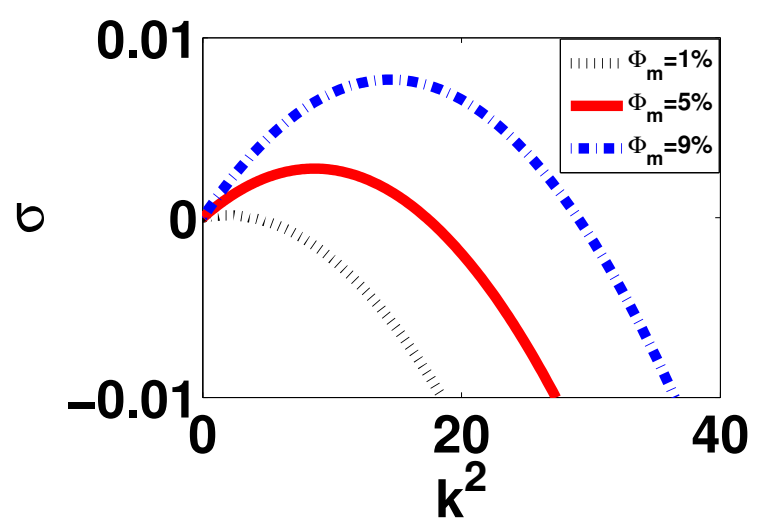

Figure 3: Plot of the growth rate $\sigma$ as a function of $k^{2}$ for $\Phi_{m}=1 \%$ (dots) line, $\Phi_{m}=5 \%$ (solid) line and $\Phi_{m}=9 \%$ (dashed) line.

We applied the Poincaré - Lindstedt method on the evolution equation (28) and obtained a uniformly valid periodic solution given by equation (39) and depicted in figure (1).

\section{References}

[1] F. Blanchette, Mixing and convection driven by particles settling in temperaturestratified ambients, Int. J. Heat Mass Transfer, 56, (2013) 732-740, DOI: 10.1016/j.ijheatmasstransfer.2012.09.042.

[2] X. Yu, T. Hsu and S. Balachandar, Convective instability in sedimentation: Linear stability analysis, Journal of Geophysical Research: Oceans, 118, (2013) 256-272, DOI: 10.1029/2012JC008255.

[3] F. Winkel, S. Messlinger, W. Schöpf, I. Rehberg, M. Siebenbürger, and M. Ballauff, Thermal convection in a thermosensitive colloidal suspension, New Journal of Physics, 12, 053003 (2010), DOI:10.1088/1367-2630/16/7/079501. 
[4] A. Ryskin and H. Pleiner, Thermal convection in colloidal suspensions with negative separation ratio, Phys. Rev. E.71, 056304 (2005), DOI: 10.1103/PhysRevE.71.056303.

[5] B.L. Smorodin, I.N. Cherepanov, B.I. Myznikova and M.I. Shliomis, Traveling-wave convection in colloids stratified by gravity,Phys. Rev. E, 84, 026305 (2011), DOI: 10.1103/PhysRevE.84.026305.

[6] M.I. Shliomis and B.L. Smorodin, Onset of convection in colloids stratified by gravity, Phys. Rev. E. 71, 036312 (2005), DOI: 10.1103/PhysRevE.71.036312.

[7] M. Glässl, M. Hilt and W. Zimmermann,Convection in colloidal suspensions with particle-concentration-dependent viscosity, Eur. Phys. J. E. 32, (2010) 265-272, DOI:10.1140/epje/i2010-10652-9.

[8] Dhananjay, G.S. Agrawal and R. Bhargava, Rayleigh-Bénard Convection in Nanofluid, Int. J. of Appl. Math and Mech. 7 (2011) 61-76,

[9] J. Buongiorno, et al., A benchmark study on the thermal conductivity of nanofluids, $J$. Appl. Phys. 106, 094312 (2009), DOI:10.1063/1.3245330.

[10] S.U.S. Choi, Z.G. Zhang, W. Yu, F.E.Lockwood, and E.A. Grulke, Anomalous thermal conductivity enhancement in nanotubes suspensions, Appl. Phys. Letters 79, 2252 (2001), DOI: 10.1063/1.1408272.

[11] Buongiorno, J.,Convective transport in nanofluids, ASME J. Heat Transfer, 128, (2006) 240-250, DOI: 10.1115/1.2150834.

[12] L. Hadji, Convection in colloidal suspensions of solid particles: A comparative study between the homogeneous mixture and the particulate medium models, Chem. Eng. Comm. 199, (2012) 1394-1411, DOI: 10.1080/00986445.2012.661378.

[13] L. Hadji, M. DarAssi,Influence of sedimentation on the threshold for Soret-driven convection in colloidal suspensions, Phys. Rev. E. 89, 013014 (2014), DOI: 10.1103/PhysRevE.89.013014.

[14] M. DarAssi, L. Hadji, Analysis of the interplay between sedimentation and thermophoresis in the presence of convection in colloidal suspensions, Proceedings of the ASME 2014 4th Joint US-European Fluids Engineering Division Summer Meeting, (2014), DOI:10.1115/FEDSM2014-21078.

[15] B. H. Chang, A.F. Mills and E. Hernandez, Natural convection of micro particles suspensions in thin enclosures, Int. J. Heat Mass Transfer 51, (2008) 1332-1341, DOI: 10.1016/j.ijheatmasstransfer.2007.11.030.

[16] J. Mewis, N. J.Wagner, Colloidal suspension rheology, Cambridge University Press, Cambridge (2012).

[17] M. Tokuyama and I. Oppenheim, Dynamics of hard-sphere suspensions, Phys. Rev. E. 50, (1994) R16, DOI: 10.1103/PhysRevE.50.R16.

[18] M. Tokuyama and I. Oppenheim, On the theory of concentrated hard-sphere suspensions, Physica A. 216, (1995) 85-119, DOI: 10.1016/0378-4371(94)00280-7.

[19] J. K. G. Dhont, Thermodiffusion of interacting colloids. I. A statistical thermodynamics approach, J. Chem. Phys. 120, 1632 (2004), DOI: 10.1063/1.1633546.

[20] J. K. G. Dhont, Thermodiffusion of interacting colloids. II. A microscopic approach, J. Chem. Phys. 120, 1642 (2004), DOI: 10.1063/1.1633547 
[21] H. Ning, J. Buitenhuis, J. K. G. Dhont, and S. Wiegandd, Thermal diffusion behavior of hard-sphere suspensions, J. Chem. Phys. 125, 204911 (2006), DOI: 10.1063/1.2400860.

[22] L. Hadji, Modeling convection onset in colloidal suspensions of particles, J. Non-Equilib. Thermodyn. 36, (2011) 203-227, DOI: 10.1515/JNETDY.2011.013.

[23] P.G.Drazin, W.H.Reid, Hydrodynamic stability, Cambridge University Press, Cambridge (2004).

[24] F. H. Busse and N. Riahi, Nonlinear convection in a layer with nearly insulating boundaries, J. Fluid Mech., 96, (1980) 243-256, DOI: 10.1017/S0022112080002091.

[25] L. Hadji and M. Schell, Transition to Soret driven convection in a system with nearly impermeable boundaries, Phys. Fluids A., 1, 1467 (1989), DOI: 10.1063/1.857324.

[26] E. Knobloch, Pattern selection in binary-fluid convection at positive separation ratios, Physical Review A,40, (1989) 1549-1559,DOI: 10.1103/PhysRevA.40.1549.

[27] C.J. Chapman, M.R.E. Proctor, Nonlinear Rayleigh-Bénard convection with poorly conducting boundaries, J. Fluid Mech., 101, (1980) 759-782, DOI: https://doi.org/10.1017/S0022112080001917.

[28] L.Hadji, Long-wavelength analysis of the coupling between solidification and Soret-driven convection at positive separation ratio, Phys. Rev. E. , 47, No. 2, (1993) 1078-1086, DOI:https://doi.org/10.1103/PhysRevE.47.1078.

[29] M.R.E. Proctor, Planform selection by finite-amplitude thermal convection between poorly conducting slabs, J. Fluid Mech., 113, (1981) 469-48, DOI: https://doi.org/10.1017/S0022112081003595.

[30] P.C. Matthews, A model for the onset of penetrative convection, J. Fluid Mech., 188, (1988) 571-583, DOI: https://doi.org/10.1017/S0022112088000850. 